\title{
Toxicon
}

Toxicon $51(2008) 457-467$

www.elsevier.com/locate/toxicon

\section{Hepatic recovery after damage produced by sub-chronic intoxication with the cyanotoxin microcystin LR ${ }^{\text {is }}$}

\author{
Darío Andrinolo ${ }^{\mathrm{a}, *}$, Daniela Sedan $^{\mathrm{b}}$, Luis Telese ${ }^{\mathrm{b}}$, Claudia Aura ${ }^{\mathrm{b}}$, Silvia Masera ${ }^{\mathrm{b}}$, \\ Leda Giannuzzi ${ }^{\mathrm{a}}$, Carlos Alberto Marra ${ }^{\mathrm{c}}$, María J.T. de Alaniz ${ }^{\mathrm{c}}$ \\ ${ }^{a}$ Centro de Investigación y Desarrollo en Criotecnología de Alimentos (CIDCA), Universidad Nacional de La Plata, La Plata, Argentina \\ ${ }^{\mathrm{b}}$ Toxicología y Química Legal, Facultad de Ciencias Exactas, Universidad Nacional de La Plata, La Plata, Argentina \\ ${ }^{\mathrm{c}}$ INIBIOLP (Instituto de Investigaciones Bioquímicas de La Plata), Cátedra de Bioquímica, Facultad de Ciencias Médicas, \\ Universidad Nacional de La Plata, La Plata, Argentina
}

Received 30 May 2007; received in revised form 5 November 2007; accepted 8 November 2007

Available online 23 November 2007

\begin{abstract}
The effect of sub-chronic exposure of intraperitoneal (i.p.) injections of microcystin-LR (MC-LR) on microscopic tissue architecture, hepatic function and lipid peroxidation has been studied in liver and kidney of mice. Mice were treated i.p. with $25 \mu \mathrm{g}$ of pure MC-LR $/ \mathrm{kg}$ body weight or saline solution for 1 month (every 2 days) with the aim of producing an inflictive stage with evident damage. Histopathological analysis of dissected livers of mice showed a disrupted lobar architecture and the development of cytoplasmatic vacuoles. According to this, a significant increase in hepatic lipid content and in lipid peroxidation levels in liver and kidney was found in MC-LR-treated animals when compared with controls. Moreover, serum alkaline phosphatase and aspartate aminotransferase activities showed a significant alteration in MC-LR-treated animals. After damage, progression or recovery was studied for 1 and 2 months of wash-out. The recovery from liver damage was evident at the cytological and physiological level, only the recovery of lobar architecture was incomplete along the period investigated. In conclusion, the present study demonstrates the ability of hepatic tissue to recover from damage produced by sub-chronic MC-LR administration. The dynamic interplay between damage and tissue-repairing response in determining the ultimate outcome of toxicity should be considered in risk-assessment studies. (C) 2007 Elsevier Ltd. All rights reserved.
\end{abstract}

Keywords: Microcystins; Sub-chronic exposure liver recovery; Lipid peroxidation

\footnotetext{
Ethical statement: All animals in this work received human care, and studies were conducted in accordance with the "Principles of Laboratory Animal Care" (National Institutes of Health Publication no. 86-23, revised 1985) and approved by the local standard for protecting animal welfare.

*Corresponding author.

E-mail address: dandrinolo@yahoo.com (D. Andrinolo).
}

\section{Introduction}

Cyanobacteria or blue-green algae are prokaryotic photosynthetic organisms that grow-and can flourish-in diverse environments including marine, brackish and fresh waters. Many species of cyanobacteria can produce toxins named cyanotoxins. They are classified into neurotoxins, hepatotoxins and skin irritants, and are distributed worldwide (Carmichael, 1992, 1994). 
Microcystins (MCs) are an important group of hepatotoxins distributed worldwide; they are produced primarily by the freshwater cyanobacterium Microcystis aeruginosa, although other genera of cyanobacteria such as Oscillatoria sp., Anabaena sp. and Cilindrospermopsis sp. can also produce them.

MCs are cyclic heptapeptides with a general structure of cyclo-(D-alanine 1-X2-D-MeAsp3-Z4Adda 5-D-glutamate 6-Mdha7) in which amino acids 2 and 4 are variable L-amino acids (DMeAsp3 is D-erythro- $\beta$-methylaspartic acid, and Mdha is $N$-methyldehydroalanine, while the amino acid Adda is $(2 S, 3 S, 8 S, 9 S)$-3-amino-9-methoxy-2,6,8-trimethyl10-phenyldeca-4,6-dienoic acid). The Adda component of MCs is responsible for their toxicity (Carmichael, 1992; Ressom et al., 1994). There are about 70 structural variants of MCs formed by the variation in all 7 amino acids, primarily the L-amino acids at positions 2, 3, 4 or 7. Different variants of MCs could be released simultaneously as a single Microcystis spp. bloom that may consist of different strains (Chorus and Bartram, 1999). Microcystin-LR (MC-LR) is the most widely studied MC variant, which has the amino acids leucine and arginine at positions 2 and 4, respectively. Also, MC-LR is the most widely distributed variant of all MCs.

After ingestion of MC-LR the absorbed peptide was cleared from plasma within an hour and transported into hepatocytes by the bile acid transport system (Robinson et al., 1991a,b). MCs inhibit specifically the activity of the phosphatases 1 and 2A (intracellular proteins involved in a wide range of metabolic pathways) by a covalent bond between the toxin and the catalytic subunit of the enzyme. In general, MCs bind to residues of cysteine (Cys) present in the enzyme and other SH groups in compounds such as reduced glutathione (GSH) (Takenaka, 2001).

Human exposure to MC-LR was primarily due to ingestion of contaminated drinking water (Gupta, 1998) and recreational contact with contaminated water. It also occurred by consumption of fish or blue-green algae products from contaminated water, or accidentally by the use of MC-LRcontaminated water as reported in Caruaru, Brazil, where renal dialysis patients exposed to MC-LR had liver failure initially, and finally death (Caruaru syndrome). Jochimsen et al. (1998) and Rao et al. (2002) reported that human exposure resulted in dermal irritation and gastroenteritis.

Acute illnesses and death in both human and animals following exposure to MC-contaminated water have been reported worldwide. Liver damage and death occur due to a fatal hepatic congestion and hypovolemic shock (Carmichael, 1994). Acute intoxication with MC-LR induces cytoskeletal alterations, apoptosis and necrosis of hepatocytes resulting in intrahepatic hemorrhage. The effect of acute exposure of intraperitoneal (i.p.) injection of MC-LR on antioxidant enzymes and lipid peroxidation has been described in liver and kidney of rats treated with high doses of MC-LR (100, 150 and $230 \mu \mathrm{g} / \mathrm{kg}$ body weight). The activities of glutathione peroxidase (GSH-Px), glutathione reductase (GR), superoxide dismutase (SOD) and catalase (CAT) in liver were significantly decreased in MC-LR-treated rats. Concomitantly, significant increases in lipid peroxidation levels were observed in liver and kidney from MC-LR-treated rats, suggesting that the oxidative stress is an important factor in the pathogenesis of MC-LR-induced toxicity (Moreno et al., 2005).

However, chronic toxicity was not sufficiently studied. This question is important since it could become an important form of exposure due to either the use of marginal water supplies or the presence of MCs in drinking water (Ueno et al., 1996; Zhou et al., 2002). Chronic intoxication studies evidenced a wide range of hepatic damage such as cytosolic vacuolation, single-cell necrosis, fibrosis and tumor promotion (Ito et al., 1997; Solter et al., 1998; Guzman and Solter, 2002). After 28 days of continuous infusion to rats of doses between 3 and $9 \mu \mathrm{g}$ of $\mathrm{MC}-\mathrm{LR} / \mathrm{kg}$ body weight, a dose-dependent increase in several serum biochemical tests, including $\gamma$-glutamyl transferase, alkaline phosphatases, sorbitol dehydrogenase, aspartate aminotransferase and bile acids was observed (Solter et al., 1998).

Chronic nephrotoxicity has also been demonstrated in rats treated with i.p. injections of MC-LR $(10 \mathrm{mg} / \mathrm{kg})$ or MC-YR $(10 \mathrm{mg} / \mathrm{kg})$ every 2 days for 8 months. Histopathological investigations revealed collapsed glomeruli with thickened basement membranes, dilated tubuli filled with eosinophilic casts and DNA damage (Milutinovic et al., 2003).

In these kinds of studies, the current practice is to measure the toxic injury at the end point, named inflictive stage or stage 1 . But it has been shown that the opposing biological dynamic events named stage 2 (progressive or regressive stage) characterized by cell division and tissue repair could play a decisive role in the outcome of hepatotoxicity (Mehendale, 1994, 1995; Mangipudy et al., 1995; Anand et al., 
2003). Through in vitro test systems, usually employed in toxicity studies, we were unable to explore time-course evolution of the compensatory tissue repair response. For this reason, the aim of this study was to determine in a murine model whether the hepatic damage produced by prolonged sub-lethal exposure to MC-LR can be reversed after sub-chronic exposure.

An inflictive stage was produced by the administration of $25 \mu \mathrm{g}$ MC-LR/kg i.p. to mice every 2 days for 1 month. Then, two groups of mice were maintained without any exposition to MC-LR for 1 and 2 months, respectively, to permit a wash-out process. Histopathological analyses in hepatic and renal tissue samples, evaluation of plasmatic markers of renal and hepatic functions, and a general analysis of lipid content in liver were performed at the end of stage 1 and after 1 and 2 months of the wash-out period.

\section{Materials and methods}

\subsection{MC-LR purification}

MC-LR was purified from natural blooms of $M$. aeruginosa collected at the Rio de la Plata basin by conventional methods (Lawton et al., 1994) with slight modifications. Briefly, cells were broken by three frozen and unfrozen cycles, and the extract was cut with chloroform/methanol $(50 / 50 \mathrm{v} / \mathrm{v})$. The aqueous fraction was concentrated in a rotovapor. Finally, the purification was performed with semi-preparative high-performance liquid chromatography (HPLC). The HPLC system was HP 1100 with a degassed module and diode array detector system. The preparative column utilized was TERMO Hyperprep HS C18 $(250 \times 10 \mathrm{~mm})$, and the mobile phase was phosphate buffer ( $\mathrm{pH} 7.0$ ) with $30 \%$ acetonitrile, run in isocratic conditions, at $5 \mathrm{ml} / \mathrm{min}$ and $\mathrm{UV}$-visible detection $(\lambda=238 \mathrm{~nm})$.

The peak corresponding to MC-LR was collected separately, concentrated and desalted with a C 18 cartridge previously activated. MC-LR was eluted with a solution of methanol-water (90-10) and the methanol was evaporated. The toxin was tested by the HPLC-MS method (Barco et al., 2002). The concentration and identification of MC-LR were performed in comparison with a commercial standard (Sigma Chemical Co., St. Louis, MO, USA). The working solution was prepared freshly by diluting the toxin in sterile saline solution.

\subsection{Mice and treatment}

Twenty-eight male mice N:NIH-S (20-22 g) were reared at the Faculty of Veterinary, National University of La Plata (Universidad Nacional de La Plata). Four animals were housed per cage in treated groups and three in control groups and given free access to food and water that were supplied daily. Animals were maintained under $12 \mathrm{~h}$ light/dark cycles and allowed to acclimatize to their surrounding conditions (well-ventilated room maintained at $23 \pm 1{ }^{\circ} \mathrm{C}$ ) for 1 week before experiments started.

All animals received human care, and studies were conducted in accordance with the "Principles of Laboratory Animal Care" (National Institutes of Health Publication no. 86-23, revised 1985) and approved by the local standard for protecting animal welfare.

Mice were divided at random into three groups. Each group had four treated animals and three controls. In all groups, the animals were treated with an i.p. injection at a dose of $25 \mu \mathrm{g} / \mathrm{kg}$ body weight of MC-LR every $48 \mathrm{~h}$ for 1 month. The toxin was diluted with saline solution $(0.9 \% ; \mathrm{w} / \mathrm{v})$ at the moment of use. All animals were injected at the same hour with the same volume of solution $(300 \mu \mathrm{l})$ and with the frequency indicated for the corresponding MC-treated group. The dose was chosen by analyzing the literature data to produce evident damage and then to observe the recovery.

On the following day after the final toxin dose was administered (on day 30 for group 1), and after 1 (group 2) and 2 months (group 3) without exposure to the toxin, mice were anesthetized with ether and a sample of blood was taken by the orbital plexus bleeding method. Mice were immediately euthanized, livers and kidneys were quickly removed, blood was washed out in ice-cold $0.9 \%$ saline solution and the collected tissues were weighed. To minimize diurnal variations, mice were routinely killed between 12:00 and 13:00 h. Tissue samples were immediately homogenized, aliquoted into Eppendorf tubes and frozen at $-80^{\circ} \mathrm{C}$ under pure nitrogen atmosphere until used for biochemical determinations.

\subsection{Histological studies}

Liver and kidney sections were fixed in 25 volumes of $10 \%$ formalin in phosphate-buffered saline (PBS), pH 7. Tissue samples were dehydrated 
in an ethanol gradient, placed in chloroform to replace the ethanol and then placed in liquid wax. Sections (4-5 $\mu \mathrm{m}$ thick) were stained with hematoxylin and eosin (H\&E) (Culling, 1975). Tricromico de Gomori (Gomori, 1950) and periodic acid staining (PAS) (Mc Manus, 1948; Bancroft and Cook, 1994) were also performed.

\subsection{Biochemical markers}

Blood was collected in heparinized tubes and immediately centrifuged at $5000 \mathrm{~g}$ for $5 \mathrm{~min}$. Plasmatic fractions were separated and analyzed for alkaline phosphatase (ALP), aspartate transaminase (AST) and alanine transaminase (ALT) levels, and urea and creatinine concentrations (commercial kits, Wiener Laboratory, Rosario, Argentina). To determine lipid peroxidation, samples from renal and hepatic homogenates were processed for thiobarbituric acid reactive substances (TBARS) determination. The fluorometric method described by Yagi (1976) was used. Total hydroperoxides (ROOHs) were measured using the technique by NouroozZadeh et al. (1995) based on the "FOX assay".

\subsection{Lipid extraction and separation}

Total lipids were extracted from the samples using the method described by Folch et al. (1957). Phospholipids and neutral lipid fractions were separated from the Folch extracts by the micro-column chromatography method described elsewhere (Hanahan et al., 1957). Organic phases containing neutral or polar lipid classes were further separated by two chromatographic procedures as described elsewhere (Malins and Mangold, 1960; Neskovic and Kostic, 1968). After development, the plates were dried under nitrogen. Lanes containing the experimental samples were covered with a glass plate, while the makers were briefly exposed to a spray of methanolic iodine $(1.5 \%, \mathrm{w} / \mathrm{v})$, or the entire plate was stained following the procedure described elsewhere (Augert et al., 1989; Nakamura and Handa, 1984). Areas of interest were scraped off the plates and the isolated lipids recovered by elution with chloroform/methanol $(1: 1, \mathrm{v} / \mathrm{v})$ (efficiency of recovery was no less than $89 \%$ for neutral lipids and $96 \%$ for phospholipids).

\subsection{Statistical methods}

All results were subjected to one-way analysis of variance (ANOVA), and they represent means $\underline{ \pm}$
SEM of 3-4 animals per group. Differences in mean values between groups were assessed by the twotailed Student's $t$-test and $p<0.05$ was considered statistically significant.

\section{Results}

\subsection{Organ weight and appearance}

Body weight gain over a 1-month period did not differ significantly in exposed groups compared with weight gain in the control ones. In concordance with this, the appearance and behavior of the mice injected with MC-LR were normal, and it was not possible to discriminate them from those of mice in control groups. The initial weight of the mice (in both control and treated groups) was $22.1 \pm 0.75 \mathrm{~g}$, and it was $29.34 \pm 0.63 \mathrm{~g}$ at the end of the inflictive stage, $33.15 \pm 0.95 \mathrm{~g}$ at the end of the first month of wash-out and $35.05 \pm 1.2 \mathrm{~g}$ at the end of the second month of wash-out (mean $\pm \mathrm{SEM} ; n=28$ ).

Normalized weight of the mice obtained as the ratio between treated animals and control ones did not show any significant difference (Fig. 1). Although the animals remained clinically normal, the aspect of livers from exposed mice at the end of the toxin administration was dark and moderately but statistically and significantly larger $(p<0.05)$ than the livers obtained from control mice (Fig. 1). Also, livers from treated animals had a reticular pattern and none of them presented hemorrhagic damage characteristic of acute lethal doses.

After the first month of the wash-out period, livers from all animals that received MC-LR recovered the normal size and macroscopic aspect.

\subsection{Serum chemistry}

Three markers of hepatic function were tested: ALP, AST and ALT. Plasmatic ALP activity showed a significant increase in MC-LR-treated animals in comparison with that in the control group at the end of the 30-day exposure period. This alteration reversed and reached normal values during the first month of wash-out. However, plasmatic levels of AST showed a significant increase only at the end of the first month of wash-out. It showed normal levels at the end of the inflictive stage, and at the end of the second month of stage 2 when compared with control levels. Values of ALT showed no change in MC-LR-treated animals in comparison with the saline-treated ones (Fig. 2a). 


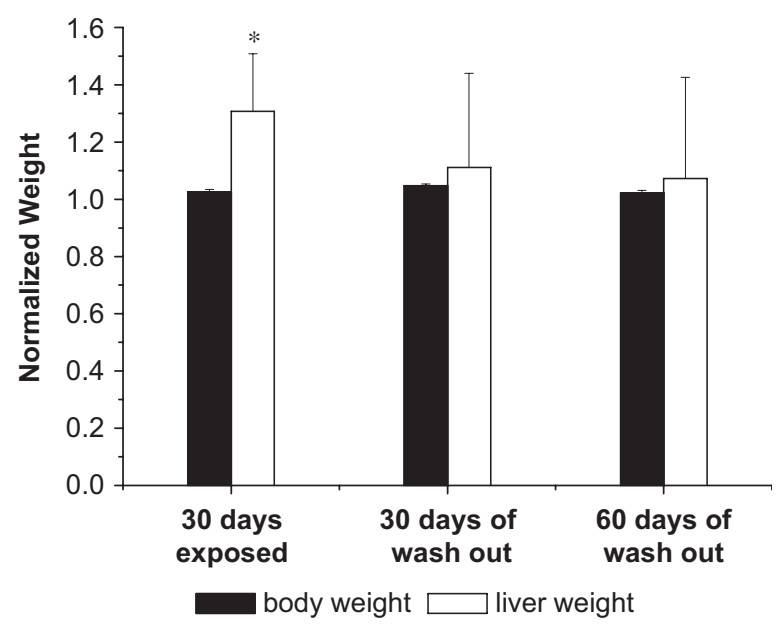

Fig. 1. Body and liver weight of mice injected with i.p. doses of $25 \mu \mathrm{g} / \mathrm{kg}$ of MC-LR (every 2 days for 30 days) analyzed at the end of stage 1 (group 1), and after 1 or 2 months of wash-out (group 2 and 3 , respectively). The values are expressed as mean of body or liver weight of treated groups normalized by their respective controls $( \pm \mathrm{SE}, n=4)$. There were no differences observed in body weight along the experiment. The liver weight ratios showed a significant increase after the inflictive stage. ${ }^{*}$ indicates results significantly different with respect to control values $(p<0.05)$.

The tested markers of renal function (creatinine and urea) were normal compared with control data along the experimental period, evidencing that the renal system was not significantly damaged (Fig. 2b).

\subsection{Histological studies}

There was no histopathological alteration in kidneys in contrast with the important damage we found in hepatic tissue from MC-treated animals. H\&E-stained liver sections showed that mice that received MC-LR for 30 days had nuclear discariosis, a high degree of binucleation and clear cytoplasmatic vacuolation with hepatic steatosis. Vacuoles were not stained with PAS methods indicating that they did not contain glycogen or glycosaminoglycan. A complete loss of liver architecture was found in mice treated with MC-LR (Fig. 3b). After 1 month of wash-out, this condition was partially reversed, and micro-vacuolation and nuclear hyperchromasia were observed (Fig. 3c). After 2 months of wash-out, liver architecture was recovered even though preserved dilated sinusoidal spaces were observed (Fig. 3d). There was no evidence for the apoptotic process. Slices did not show shrunken hepatocytes, hypereosinophylic cy-
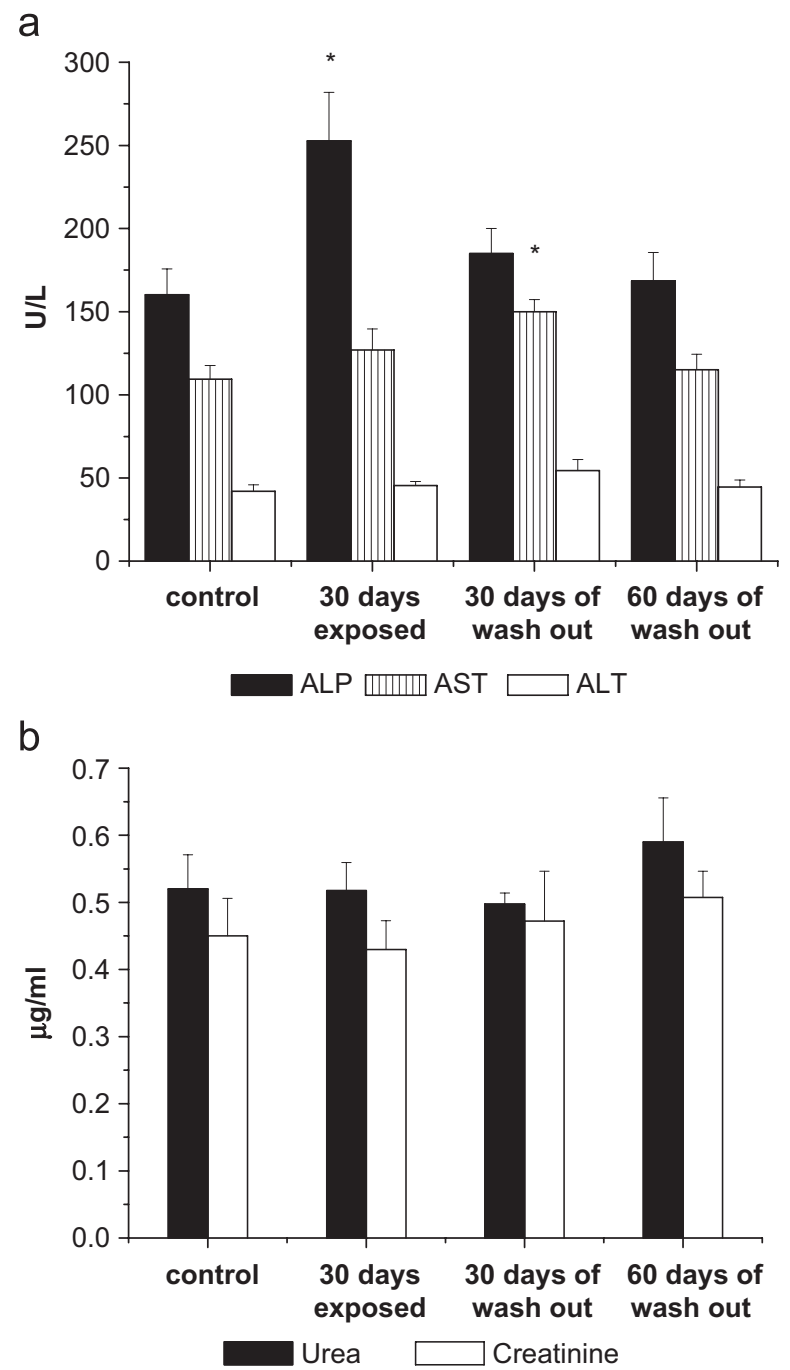

Fig. 2. Hepatic and renal function markers are shown. (a) Plasma levels of ALP, AST and ALT at the end of stage 1, and after 1 and 2 months of wash-out. (b) Plasma levels of creatinine and urea, (mean $\pm \mathrm{SE}, n=4)$. The standard error of each mean is shown by bars. ${ }^{*}$ represents a significant difference in control values according to ANOVA test of two independent populations $(p<0.05 \%)$.

toplasm surrounded by clear spaces and/or nuclei fragmented into small picnotic bodies. Apoptotic bodies were also absent. Livers from control animals had normal appearance with a clear cell radial arrangement surrounding the central vein (Fig. 3a).

To observe the hepatic structure and particularly the wall of the hepato-portal system the slices were stained by the trichromic method of Gomori. The walls of hepatic vessels were not disrupted and there was no evidence for any hemorrhagic process as 

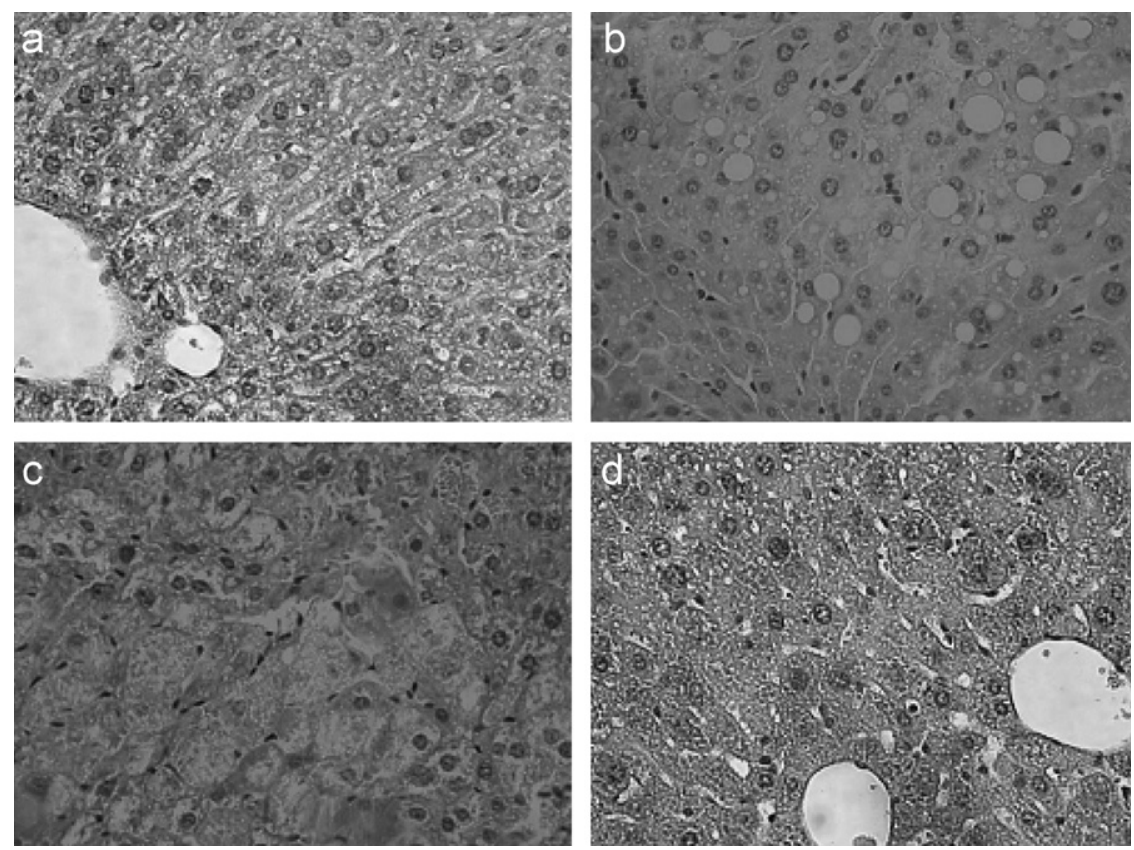

Fig. 3. Representative slices H\&E-stained $400 \times$ of liver from (a) mice treated with saline solution for 30 days that showed the normal pattern of liver parenchyma, (b) with $25 \mu \mathrm{g} / \mathrm{kg}$ MC-LR every 2 days for a month, (c) 1 month of wash-out and (d) 2 months of wash-out.

observed in acute lethal doses. The connective tissue made a dense ring around the vessel and was distributed homogenously in the parenchyma of the liver of control mice (Fig. 4a), but it was clearly reduced in liver from mice treated with MC-LR for 30 days (Fig. 4b). These conditions remained unchanged within 30 and 60 days of wash-out (Fig. 4c and d).

\subsection{Lipid peroxidation}

Lipid peroxidation induced by MC-LR treatment was determined by TBARS in hepatic and renal homogenates (Fig. 5a). In liver, a 2.5-fold increase was found in TBARS compared with the salinetreated (control) mice. This condition was reverted after 1 month of the wash-out period and completely normalized after the second month. The pattern observed in kidney homogenates was very similar, though a significant elevation of TBARS was detected even after 1 month of toxin wash-out. In agreement with the conclusions obtained by analyzing TBARS results, lipid hydroperoxide amounts determined by the FOX assay were elevated in liver and kidney of MC-LR-exposed animals compared with the control ones (Fig. 5b). Restoration of normal levels was achieved in liver and kidney after 1 or 2 months of the wash-out period. By comparing the TBAR and FOX assay determinations we observed, as in other biological samples such as plasma, that the level of ROOHs was slightly higher than that of TBARS. Notwithstanding, conclusions regarding the relative impact of the toxin treatment on lipid peroxidation in kidney with respect to liver $(124 \%$ and $112 \%$ changes, respectively) were also confirmed by $\mathrm{ROOH}$ measurements since restoration to control data was achieved in kidney 1 month later than in liver.

\subsection{Sub-chronic effects of MC-LR exposure on hepatic lipid content in mice}

In agreement with the cytoplasmatic vacuolation found after 1 month of exposure, analysis of lipid content of liver from mice exposed to MC-LR revealed a significant increase in total lipid content compared with liver from non-exposed mice. This fact remained after 1 month of wash-out and returned to basal conditions after 2 months of the wash-out period (Table 1). After 1 month of exposure, there was an increase in total lipid due to increments in both neutral and polar lipids. However, after the first month of wash-out, polar lipids returned to control values while the neutral ones maintained elevated levels. After 2 months of 

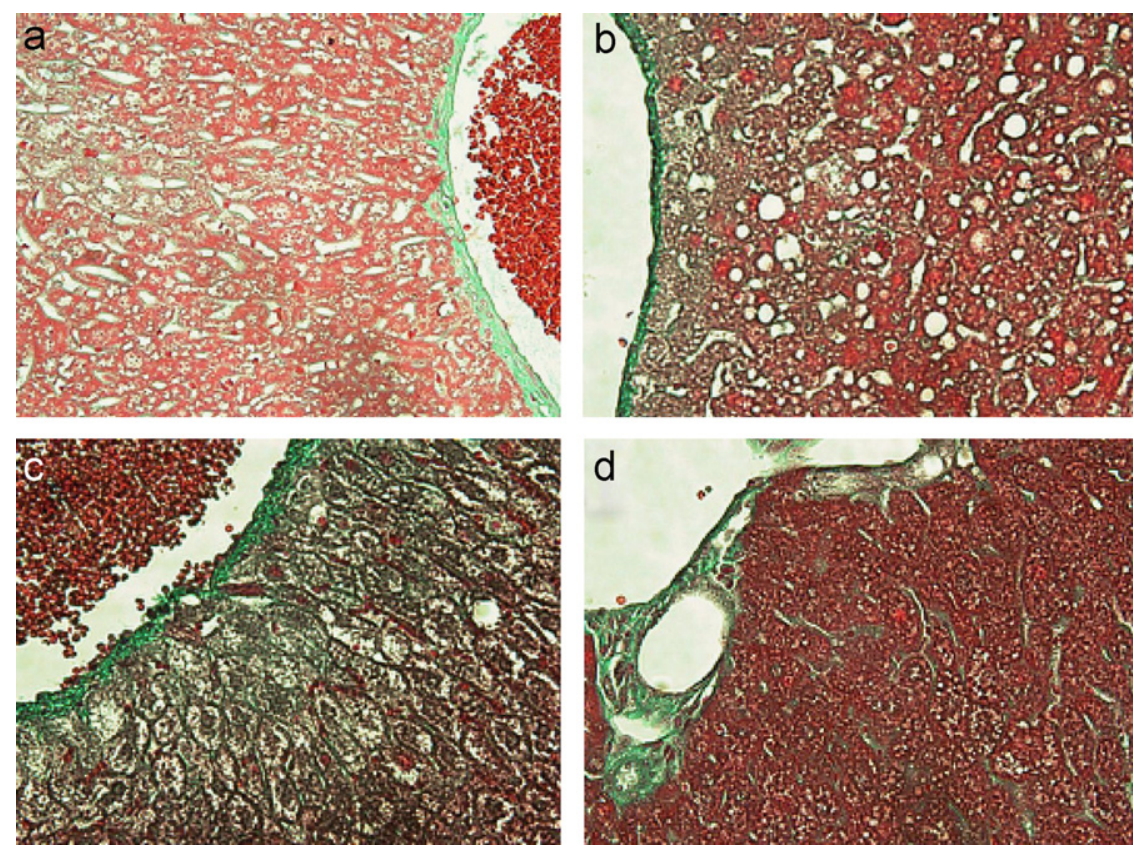

Fig. 4. Representative slices of Gomori-stained liver $(400 \times)$ are shown: (a) slices of liver from control mice showed connective tissue regularly distributed through hepatic parenchyma, forming a dense ring around the hepatic vein. (b) Representative hepatic slices from one mouse at the end of the inflictive stage with a significant loss of connective tissue in both parenchyma and vein wall. The cells had clear and large vacuoles. (c) Slice of liver from mouse after the first month of wash-out. The cells had different shape, size and vacuoles became "micro" vacuoles. Scarce connective tissue was observed. (d) Slice from mouse after 2 months of wash-out. Cells bigger than control ones and nucleus with irregular morphology were observed. The hepatic tissue showed an important degree of recovery with diminution of vacuolation and appearance of sinusoids. Connective tissue was scarce.

wash-out, the levels of both polar and neutral lipids were completely restored. These changes caused the initial increase of the polar lipids/neutral lipids $(\mathrm{PL} / \mathrm{NL})$ ratio and its subsequent normalization.

\section{Discussion}

In this study, mice were exposed to i.p. administration of 15 doses of $25 \mu \mathrm{g} / \mathrm{kg}$ of MC-LR (every 2 days for 1 month) with the aim of producing significant damage; then the regression of the damage was observed.

The doses and time of sub-chronic exposure used in this study did not cause any death or sign of clinical illness in mice. No evidence of renal toxicity was observed either histologically or on clinical chemistry examination. In agreement with previous studies in a rat model (Guzman and Solter, 2002), body weights were not different from control ones, indicating that this index cannot be used as a sign of sub-chronic exposure to MC-LR. Also, it may explain why sub-chronic intoxication of humans and animals is currently not detected.
At the same time, hepatic integrity was seriously affected at the end of the inflictive stage. Hepatic lesions were in agreement with other studies, including hepatocellular disarray and loss of hepatic architecture with binucleation, karyomegaly, cytoplasmatic vacuolation, steatosis and hypertrophic hepatocytes.

Histological evaluation of liver specimens and biochemical data demonstrated that following a sub-chronic exposure to MC-LR the mice developed hepatic steatosis, as rats do. This was reverted when the exposure ended.

Hepatocyte apoptosis has been described as a response to acute exposure in mice (Yoshida et al., 1997), rats (Solter et al., 1998) and trout (Fischer et al., 2000). The high susceptibility of hepatocytes to undergo programmed cell death with sub-lethal doses of MC-LR appeared to be an early response to intoxication detectable $24 \mathrm{~h}$ after an acute exposure. Nevertheless, apoptosis became rare in mice that received sub-lethal doses for 7 days (Guzman and Solter, 2002). According to this, and to the idea that a regenerative phase started in liver 

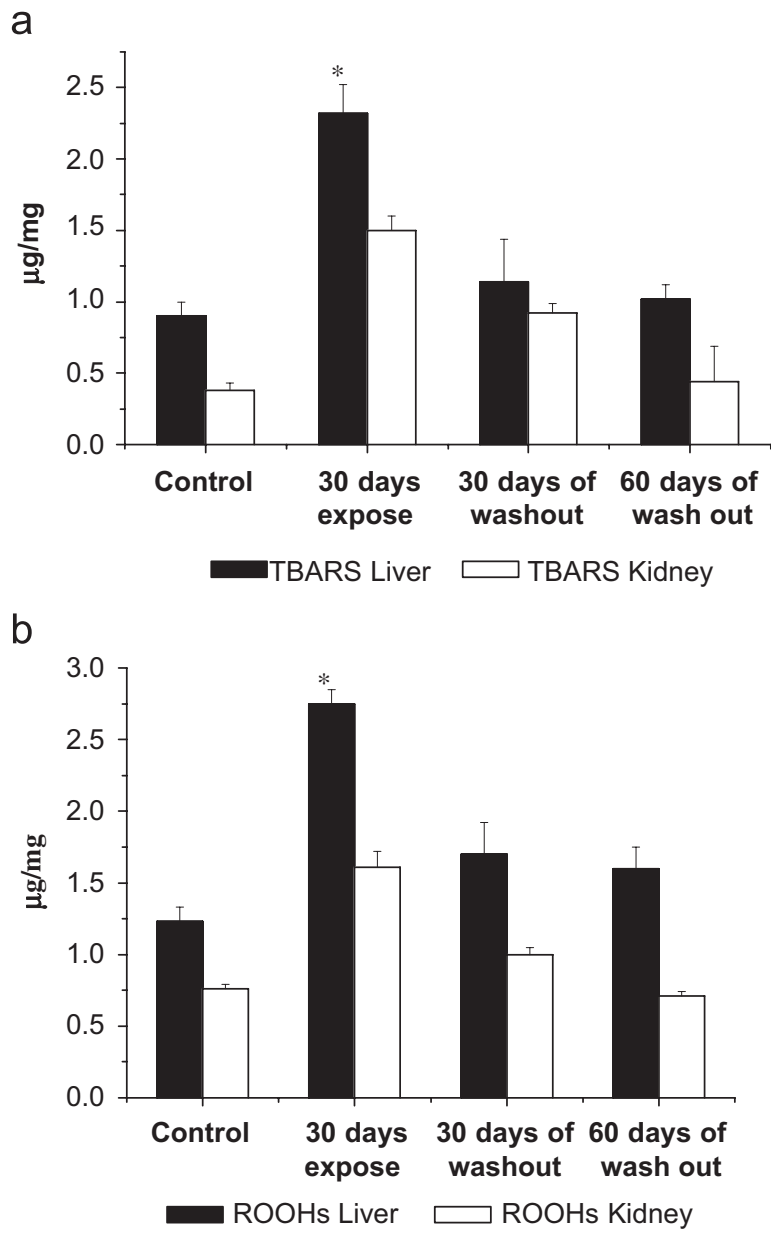

Fig. 5. Lipids were determined as described in Section 2. Results were the mean $(n=4, \pm 1 \mathrm{SD}$ of the mean), and they were expressed as $\mu \mathrm{g} / \mathrm{mg}$ tissue protein. ${ }^{*}$ indicates results significantly different with respect to control values $(p<0.05)$.

after apoptosis, in our study no apoptotic bodies were detected after 1 month of the inflictive stage.

On the other hand, the tumor-promoting activity of $\mathrm{MC}$ is likely to arise as a consecuence of its ability to potently inhibit PP2A, which regulates several mitogen-activated protein kinases (MAPKs) The MAPKs in turn regulate transcription of genes required for cellular proliferation, such as the transcription activator protein c-Jun. In addition, oxidative stress is a known stimulus of MAPK and $\mathrm{H}_{2} \mathrm{O}_{2}$ can induce the activity of JNK. This effect was evidenced in experiments in which after 100 i.p. injections over a period of 28 weeks of a sub-lethal dose $(20 \mu \mathrm{g} / \mathrm{kg})$ of MC-LR, neoplastic nodules were observed without the use of an initiator (Ito et al., 1997). Considering the similarity between the applied dose, the supplying method, the frequence and the model animal used by Ito et al. (1997) and those used for this work, it may be said that the exposure time used for this work would be insufficient to observe neoplastic developments.

Increased serum activities of enzymes considered as biomarkers for hepatocellular damage have been described in acute toxicity studies (Hermansky et al., 1991; Runnegar and Falconer, 1982). Some other studies have been successful in detecting increased serum ALT activity following prolonged MC-LR exposure (Falconer et al., 1983, 1988). The findings of this current study and previous studies suggest that serum ALT activity may lack sensitivity as a clinical marker of prolonged sub-lethal MC-LR exposure.

In chronic studies using mice, Solter et al. (1998) found a significant increase in several serum biochemical tests including $\gamma$-glutamyl transferase, alkaline phosphatases, sorbitol dehydrogenase, aspartate aminotransferase and bile acids. These changes were observed in i.p. doses of $9 \mu \mathrm{g}$ of MC-LR/kg body weight infused continuously for 28 days. We also demonstrated significant variations in serum biochemical parameters due to the effects of MC-LR after prolonged sub-lethal exposure. ALP activity was significantly increased with respect to control values at the end of the inflictive stage, and it returned to control levels during the wash-out period. In agreement with Solter et al. (1998) the values were within the range of 45-220 U/L, which is the normal serum level in mice.

Similar behavior was observed for serum AST activity that showed a significant increase only at the end of the first month of wash-out, while ALT activities displayed no significant changes. Because ALT is more specific to hepatocytes than several other clinical markers, such as ALP and AST, increased serum ALT activity is an important clinical indicator of hepatocellular injury. However, serum ALT activity is an insensitive marker of subchronic exposure to the hepatotoxin MC-LR in mice as well as in rats (Solter et al., 1998).

Our results indicated that the enzymes studied in this work (ALP, AST and ALT) are not appropriate biological markers for chronic exposure to MC-LR.

Exposure to MCs caused oxidative cellular damage. As in previous reports (Solter et al., 1998; Ding, 1998) we found that lipid peroxidation is one of the main manifestations of oxidative damage induced by MC-LR. Oxidative cellular damage was found to occur in both liver and kidney with similar intensity and temporal evolution, as judged 
Table 1

Major lipids classes in total homogenates from liver of control and exposed mice

\begin{tabular}{|c|c|c|c|c|c|c|}
\hline & \multirow{2}{*}{\multicolumn{2}{|c|}{$\frac{\text { Treatment }}{30 \text { days }}$}} & \multicolumn{4}{|l|}{ Wash-out } \\
\hline & & & \multicolumn{2}{|l|}{30 days } & \multicolumn{2}{|l|}{60 days } \\
\hline & Control & Exposed & Control & Exposed & Control & Exposed \\
\hline Total lipids ${ }^{\mathrm{a}}$ & $138.1 \pm 6.6$ & $189.5 \pm 9.0^{*}$ & $96.5 \pm 8.3$ & $206.0 \pm 17.0^{*}$ & $122.5 \pm 4.9$ & $138.5 \pm 6.6$ \\
\hline Polar lipids ${ }^{\mathrm{b}}$ & $76.3 \pm 2.9$ & $113.8 \pm 4.1^{*}$ & $81.0 \pm 3.0$ & $95.3 \pm 4.0$ & $73.3 \pm 2.1$ & $80.2 \pm 3.6$ \\
\hline Neutral lipids ${ }^{c}$ & $53.5 \pm 4.4$ & $60.7 \pm 2.6^{*}$ & $55.1 \pm 3.0$ & $65.7 \pm 4.5^{*}$ & $55.9 \pm 3.5$ & $53.6 \pm 4.0$ \\
\hline $\mathrm{PL} / \mathrm{NL}$ & $1.43 \pm 0.11$ & $1.87 \pm 0.07$ & $1.47 \pm 0.06$ & $1.45 \pm 0.08$ & $1.31 \pm 0.1$ & $1.49 \pm 0.08$ \\
\hline
\end{tabular}

Significant differences between exposed animals and the corresponding control group were indicated with an asterisk $(p<0.05)$.

Each sample was collected and separately analyzed under the conditions described in the experimental part. Results are the mean of four independent determinations assayed in duplicate $\pm \mathrm{SD}$, and they are expressed as

${ }^{\mathrm{a}} \mathrm{pg} / \mathrm{mg}$ protein.

${ }^{\mathrm{b}}$ pmol inorganic phosphate/mg protein.

${ }^{\mathrm{c}}$ pmol tripalmitine/mg protein.

by the lipid peroxidation biomarkers (TBARS and ROOHs). The classical evaluation of lipid peroxidation by means of the TBARS reaction should be correlated with a more specific biomarker. For this reason, we chose the FOX assay, which is able to detect the different classes of authentic hydroperoxides (ROOHs) since it is more accurate than other methods (Nourooz-Zadeh et al., 1995). Comparative studies performed in other laboratories support the view that ROOH measurements performed with this assay are more useful than other techniques in assessing oxidative stress (Nourooz-Zadeh et al., 1995). Our findings were interesting since they could be indicators of an augmented susceptibility of kidney, even in the absence of histopathological or physiological alterations due to the toxic action of MC-LR.

The present study also shows that the lipid peroxidation stimulated by sub-chronic exposure to MCs can be reverted when the exposure concludes. Concerning liver, our results were in agreement with those previously reported for rat hepatocytes (Ding et al., 1998) and rat liver microsomes (Hermansky et al., 1991). Also, they support experimental evidence suggesting a central role played by oxidative stress in the mammalian pathogenesis of MC-LR (Ding et al., 1998; Hermansky et al., 1991). As previously stated by Fawell et al. (1999) liver is the primary target for the toxin action of MC-LR. Recently, Moreno et al. (2005) reported that the acute exposure of rats to MC-LR also promoted renal alterations since kidney is involved in the elimination of MCs. However, they observed that lipid peroxidation was more evident in liver than in kidney. Interestingly, when we compared these results with those obtained in the present study it may be suggested that sub-chronic treatment with MC-LR could evoke a secondary oxidative damage in kidney tissues, whose extension was at least equivalent to that observed in liver. A prolonged exposure to the toxin justifies these results. The different responses obtained are likely to be the consequence of metabolic characteristics of the animals employed in the experiments (mouse and rat). Based on this fact, another interesting question has arisen about the duration and/or doses of MC-LR, and perhaps the inter-species differences that would be key factors to determine the real impact of MC intoxication. This may include not only pathological consequences but also tissue specificity of this toxic effect. To clarify this point, a multi-approach study regarding sub-chronic exposure of mice to $\mathrm{MC}$, and its consequences on lipid metabolism and the antioxidant defense system, is currently under study in our laboratory.

Analysis of liver lipids showed a significant increase of total lipid quantities in agreement with the appearance of lipid vacuoles. This abnormal lipid accumulation could indicate an MC-LRspecific action on lipid metabolism. The specific nature of the moieties involved and the metabolic route modified by the intoxication are matters that still remain to be further investigated.

One of the key questions in this study is the demonstration that hepatic tissue can be recovered from damage produced by sub-chronic MC-LR administration. The balance between damage and the ability of tissues to be recovered after intoxication 
may determine the ultimate outcome of toxicity. Both factors should be taken into account when considering risk-assessment studies.

The natural exposure of wild and domestic animals and humans to sub-lethal levels of MCs is likely to be unnoticed due to the absence of clinical symptoms and the lack of biological specific markers. The results of this study are essential to determine the toxicological consequences of a sublethal exposure to these toxins. The considerable degree of recovery observed within the 2 months of the wash-out period in highly affected livers indicates that a total recovery could be reached after a sub-chronic intoxication.

\section{Acknowledgments}

We wish to thank Joseph Caixach and Mónica Barco from Laboratori d'Espectrometria de Masses, Departament d'Ecotecnologies, Barcelon, Spain, for their help with the characterization of the purified toxin.

This study was supported by the Consejo Nacional de Investigaciones Científicas y Tecnológicas (CONICET), the Comisión de Investigaciones Científicas de la Provincia de Buenos Aires (CIC) and the Universidad Nacional de La Plata (UNLP), Argentina.

\section{References}

Anand, S.S., Murthy, S.N., Vishal, V.S., Mumtaz, M.M., Mehendale, H.M., 2003. Tissue repair plays pivotal role in final outcome of supra-additive liver injury after chloroform and allyl alcohol binary mixture. Food Chem. Toxicol. 41, 1123.

Augert, G., Bocckino, S.B., Blackmore, P.F., Exton, J.H., 1989. Hormonal stimulation of diacylglycerol formation in hepatocytes. Evidence for phosphatidylcholine breakdown. J. Biol. Chem. 264, 21689-21698.

Bancroft, J.D., Cook, H.C., 1994. Manual of Histological Techniques and their Diagnostic Application. Churchill Livingstone, Edinburg.

Barco, M., Rivera, J., Caixach, J., 2002. Analysis of cyanobacterial hepatotoxins in water samples by microbore reversedphase liquid chromatography-electrospray ionisation mass spectrometry. J. Chromatogr. A 959, 103-111.

Carmichael, W.W., 1992. Cyanobacteria secondary metabolites - the cyanotoxins. J Appl. Bacteriol. 72, 445-459.

Carmichael, W.W., 1994. The toxins of cyanobacteria. Sci. Am. 270 (1), 78-86.

Chorus, I., Bartram, J., 1999. In: Chorus, I., Bartram, J., (Eds.), Toxic Cyanobacteria in Water: A Guide to Their Public Health Consequences, Monitoring and Management. Published on behalf of WHO by F \& FN Spon, London.
Culling, C.F.A., 1975. Handbook of Histological Techniques, third ed. Butterworths, London.

Ding, W., Shen, H., Zhu, H., Ong, Ch., 1998. Studies on oxidative damage induced by cyanobacteria extract in primary cultured rat hepatocytes. Environ. Res. A 78, 12-18.

Falconer, I.R., Beresford, A.M., Runnegar, M.T., 1983. Evidence of liver damage by toxin from a bloom of the blue-green alga, Microcystis aeruginosa. Med. J. Aust. 1, 511-514.

Falconer, I.R., Smith, J.V., Jackson, A.R., Jones, A., Runnegar, M.T., 1988. Oral toxicity of a bloom of the cyanobacterium Microcystis aeruginosa administered to mice over periods up to 1 year. J. Toxicol. Environ. Health 24, 291-305.

Fawell, J.K., Mitchell, R.E., Everett, D.J., Hill, R.E., 1999. The toxicity of cyanobacterial toxins in the mouse: I microcystinLR. Hum. Exp. Toxicol. 18 (3), 162-167.

Fischer, W., Hitzfeld, B., Tencalla, F., Eriksson, J., Mikhailov, A., Dietrich, D., 2000. Microcystin-LR toxicodynamics, induced pathology and immunohistochemical localization in livers of blue green algae exposed rainbow trout (Oncorkynchus mykiss). Toxicol. Sci. 54, 365-373.

Folch, J., Lees, M., Stanley, G.A.S., 1957. A simple method for the isolation and purification of total lipids from animal tissues. J. Biol. Chem. 226, 497-509.

Gomori, G., 1950. A rapid one-step thrichrome stain. Am. J. Clin. Pathol. 20, 661.

Gupta, S., 1998. Cyanobacterial toxins: microcystin-LR. In Guidelines for Drinking-water Quality, Addendum to vol. 2 , Health Criteria and Other Supporting Information. World Health Organization, Geneva, pp. 95-110.

Guzman, R., Solter, P., 2002. Characterization of sublethal microcystin-LR exposure in mice. Vet. Pathol. 39, 17-26.

Hanahan, D.J., Dittnar, J.C., Warashima, E., 1957. A column chromatographic separation of classes of phospholipids. J. Biol. Chem. 228, 685-690.

Hermansky, S., Stohs, S., Elden, Z., Roche, V., Mereish, K., 1991. Evaluation of potential chemoprotectants against microcystin-LR hepatotoxicity in mice. J. Appl. Toxicol. 11, 65-74.

Ito, E., Kondo, F., Terao, K., Harada, K., 1997. Neoplastic nodular formation in mouse liver induced by repeated intraperitoneal injections of microcystin-LR. Toxicon 35 (9), 1453-1457.

Jochimsen, E.M., Carmichael, W.W., An, J.S., Cardo, D.M., Cookson, S.T., Holmes, C.E.M., Antunes, M.B.C., MeloFilho, D.A., Lyra, T.M., Barreto, V.S.T., Azevedo, S.M.F.O. Jarvis, W.R., 1998. Liver failure and death after exposure to microcystins at a haemodialysis center in Brazil. New Engl. J. Med. 33, 873-878.

Lawton, L., Edwards, C., Codd, G.A., 1994. Extraction and high-performance liquid chromatographic method for the determination of microcystins in raw and treated waters. Analyst 119, 1525-1530.

Malins, D.C., Mangold, H.K., 1960. Analysis of complex lipid mixtures by thin layer chromatographic and complementary methods. J. Am. Oil Chem. Soc. 37, 576-582.

Mangipudy, R.S., Chanda, S., Mehendale, H.M., 1995. Hepatocellular regeneration: key to thioacetamide autoprotection. Pharmacol. Toxicol. 77, 182.

Mc Manus, J.F.A., 1948. Histological and histochemical uses of periodic acid. Stain Technol. 23, 99-108.

Mehendale, H.M., 1994. Amplified interactive toxicity of chemicals at nontoxic levels: mechanistic considerations and 
implications to public health. Environ. Health Perspect. 102, 139.

Mehendale, H.M., 1995. Toxicodynamics of low level toxicant interactions of biological significance: inhibition of tissue repair. Toxicology 105, 251.

Milutinovic, A., Zivin, M., Zorc-Pleskovic, R., Sedmak, B., Suput, D., 2003. Nephrotoxic effects of chronic administration of microcystins-LR and -YR. Toxicon 42, 281-288.

Moreno, I., Pichardo, S., Jos, A., Gomez-Amores, L., Mate, A., Vazquez, C.M., Camean, A.M., 2005. Antioxidant enzyme activity and lipid peroxidation in liver and kidney of rats exposed to microcystin-LR administered intraperitoneally. Toxicon 45, 395-402.

Nakamura, K., Handa, S., 1984. Coomassie brilliant blue staining of lipids on thin-layer plates. Anal. Biochem. 142, 406-410.

Neskovic, N.M., Kostic, D.M., 1968. Quantitative analysis of rat liver phospholipids by a two-step thin-layer chromatography procedure. J. Chromatagr. 35, 297-300.

Nourooz-Zadeh, J., Tajaddimi-Sarmandi, J., McCarthy, S., Betteridge, D.J., Wolff, S.P., 1995. Elevated levels of authentic plasma hydroperoxides in NIDDM. Diabetes 44, 1054-1058.

Rao, P.V., Gupta, N., Bhaskar, A.S., Jayaraj, R., 2002. Toxins and bioactive compounds from cyanobacteria and their implications on human health. J. Environ. Biol. 23, 215-224.

Ressom, R., et al., 1994. Health Effects of Toxic Cyanobacteria. National Health and Medical Research Council, Commonwealth of Australia, Canberra (ISBN 064432908 4).

Robinson, N.A., Pace, J.G., Matson, C.F., Miura, G.A., Lawrence, W.B., 1991a. Tissue distribution, excretion and hepatic biotransformation of MCLR in mice. J. Pharmacol. Exp. Ther. 256 (1), 176-182.

Robinson, N.A., Matson, C.F., Pace, J.G., 1991b. Association of microcystin-LR and its biotransformation product with a hepatic-cytosolic protein. J. Biochem. Toxicol. 6 (3), 171-180.

Runnegar, M.T.S., Falconer, I.R., 1982. The in vivo and in vitro biological effects of the peptide hepatotoxin from the blue green alga Microcystis aeruginosa. South Afr. J. Sci. 78, 363-366.

Solter, P., Wollenberg, G., Huang, X., Chu, F., Runnegar, M., 1998. Prolonged sublethal exposure to the protein phosphatase inhibitor, microcystin-LR results in multiple dosedependant hepatotoxic effects. Toxicol. Sci. 44, 87-96.

Takenaka, S., 2001. Covalent glutathione conjugation to cyanobacterial hepatotoxin microcystin LR by F344 rat cytosolic and microsomal glutathione $S$-transferases. Environ. Toxicol. Pharmacol. 9, 135-139.

Ueno, Y., Nagata, S., Tsutsumi, T., Hasegawa, A., Watanabe, M.F., Park, H., Chen, G.C., Chen, G., Yu, S., 1996. Detection of microcystins, a blue-green algal hepatoxin, in drinking water sampled in Haimen and Fusui, endemic areas of primary liver cancer in China, by highly sensitive immunoassay. Carcinogenesis 17, 1317-1321.

Yagi, K., 1976. A simple fluorimetric assay for lipoperoxide in blood plasma. Biochem. Med. 15, 212-216.

Yoshida, T., Makita, Y., Nagata, S., Tsutsumi, T., Yoshida, F., Sekijima, M., Tamura, S., Ueno, Y., 1997. Acute oral toxicity of microcystin-LR a cyanobacterial hepatotoxin, in mice. Nat. Toxins 5, 91-95.

Zhou, L., Yu, H., Chen, K., 2002. Relationship between microcystin in drinking water and colorectal cancer. Biomed. Environ. Sci. 15 (2), 166-171. 